\title{
Severe akathisia as a side effect of metoclopramide
}

\author{
Arthur R. Van Gool · Jeanette K. Doorduijn • \\ Caroline Seynaeve
}

Received: 21 May 2010/Accepted: 4 October 2010/Published online: 30 October 2010

(c) The Author(s) 2010. This article is published with open access at Springerlink.com

\begin{abstract}
Case description A case of severe metoclopramide-induced akathisia in a breast cancer patient being treated with chemotherapy is presented, eventually culminating in hospital admission. In retrospect, this adverse effect was not recognized for several weeks because the prescription had not been properly recorded in the chart, the patient initially denied using the drug, and extensive psychological adjustment difficulties were also present. Conclusion Movement disorders as an adverse effect of metoclopramide have been described on a regular basis over the past decades. Case reports such as this confirm there is under-recognition of adverse effects and emphasize the need to take a comprehensive medication history and recognize well known side effects of medications such as metoclopramide.
\end{abstract}

Keywords Adverse drug reaction - Akathisia . Metoclopramide

A. R. Van Gool $(\bowtie)$

Yulius Academy, Yulius Mental Health, Mathenesserlaan 202, 3314 HH Rotterdam, The Netherlands

e-mail: a.vangool@yulius.nl; a.vangool@erasmusmc.nl

J. K. Doorduijn

Department of Haematology, Erasmus University Medical

Center, PO Box 2040, 3000 CA Rotterdam, The Netherlands

C. Seynaeve

Department of Medical Oncology, Erasmus University Medical Center, PO Box 2040, 3000 CA Rotterdam, The Netherlands

A. R. Van Gool

Department of Psychiatry, Erasmus University Medical Center, PO Box 2040, 3000 CA Rotterdam, The Netherlands

\section{Impact of findings on clinical practice}

- There is under-recognition of movement disorders as a side effects of metoclopramide by clinicians.

- Patients who are prescribed metoclopramide should be educated not only about its efficacy and relative safety, but also about movement disorders as a potential side effect.

- Metoclopramide-induced movement disorders are easily missed in medically complex cases, especially when other explanations seem likely to the clinician.

\section{Introduction}

Nausea and vomiting are frequently occurring complaints that can present acutely, e.g. with migraine attacks, postsurgery or during chemotherapy for cancer, or as a part of chronic conditions such as medically unexplained abdominal symptoms ("dyspepsia") or diabetic gastroparesis. As a consequence, anti-emetic drugs are frequently prescribed, both in general practice and in the hospital.

With one of these anti-emetics, metoclopramide, extensive experience has been acquired in the course of several decades, during which time it has become regarded as generally effective and safe. Furthermore, reassuring data were recently published regarding its safety in the first trimester of pregnancy [1].

However, metoclopramide is a peripherally and centrally acting dopamine (DA) antagonist and is therefore associated with the occurrence of sometimes severe psychomotor adverse effects [2,3]. Although reports specifically on metoclopramide-induced psychomotor adverse 
effects have been available for a considerable time, during the same period reports of under-recognition of these adverse effects by clinicians appeared [4-6]. Possibly in relationship with under-recognition, the Netherlands Pharmacovigilance Centre recently reported having received only 12 reports on metoclopramide-induced dystonias and tardive dyskinesias [7].

Several factors contribute to this under-recognition, including lack of awareness of clinicians and failure to make specific inquiries with patients, especially if metoclopramide is administered on an outpatient basis. In addition to this, situations can arise in which other explanations such as evident difficulties coping with disease seem likely to the clinician or when clinicians fail to explicitly state a differential-diagnosis that incorporates the side effects of drugs.

In this case, the patients' distress was initially attributed to an adjustment disorder but eventually metoclopramideinduced akathisia was diagnosed. Making a diagnosis was also difficult, due to the lack of proper documentation of the prescription of metoclopramide. This might have been related to the use of this drug as a routine for chemotherapeutic regimens.

\section{Case description}

A 58-year-old woman, divorced and living with two children aged around 18 years, was diagnosed with locally advanced breast cancer 2 years before the current event. Primary treatment consisted of chemotherapy, mastectomy and locoregional radiotherapy. During follow-up, the oncologists repeatedly recorded various somatic complaints without organic substrate as well as psychological distress.

Four months before the event, the patient was diagnosed with metastatic disease consisting of liver and bone metastases and CMF-chemotherapy, containing cyclophosphamide, methotrexate and 5-fluorouracil, in combination with trastuzumab was initiated. At the time of psychiatric referral, the first part of the third course of CMF-chemotherapy had been administered.

After the diagnosis of recurrent metastatic disease, and before the start of chemotherapy, the patient was referred to the Psycho-oncology Service, for additional psychological support. There she was considered to be overwhelmed by the bad news of the recurrence of the cancer for which there was no curative treatment option anymore, to have limited coping abilities, a tendency to react passively, and restricted abilities to benefit from psychotherapy. She had been using oxazepam and temazepam for a considerable time period. Also, some 15 years ago, she had been admitted to a psychiatric facility for unspecified problems.
Four weeks before hospital admission, she presented vague complaints of "restlessness" to her GP and her oncologist which were attributed to "hyperventilation".

The weekend immediately preceding admission, she frequently phoned the GP and the oncology department of the hospital and was finally seen by the oncologist on call. She complained of restlessness and of insomnia for several days. Physical examination and laboratory tests were unremarkable. After various fruitless efforts to reassure her, she was admitted to the oncology ward. For purposes of sedation, pipamperon $40 \mathrm{mg}$ was added to the benzodiazepines already used and a psychiatric referral was arranged for the following day.

During psychiatric consultation, the patient was seen pacing up and down the corridor of the oncology ward. When spoken separately, she was unable to remain seated on a chair and she kept walking up and down the room: a classic presentation of akathisia. In addition to this, the general impression was one of severe suffering: she was clingy and dependent, appeared both desperate and dysphoric, and taking a history was only possible to a limited degree as the patient constantly iterated: "I want other medication", "Don't you have other medication for me" and "I can't stand this any longer". She performed well on simple tests for registration, recall and concentration, her consciousness was not impaired, she was not grossly incoherent, denied having delusions or hallucinations, her mood was difficult to assess but was probably best described as despondent, and she denied suicidal ideation.

She denied having a history of anxiety disorders such as panic attacks or phobias and denied excessive alcohol use. After having lost her own mother to cancer 40 years ago, she had always been afraid of developing and dying of cancer.

In spite of the unmistakable clinical picture of akathisia, initially, we considered an adjustment disorder as she denied using drugs other than benzodiazepines and paracetamol. Only after repeated questioning and additional checking with different outpatient pharmacies, it became clear that at the initiation of CMF-chemotherapy she had been prescribed a large quantity of metoclopramide suppositories $(20 \mathrm{mg})$ to use in case of nausea or vomiting. For reasons that she could not clarify, she had begun using these suppositories on a regular basis 2-4 times a day over the past weeks. Probably because prescribing metoclopramide was routinely done at the start of chemotherapy, no entry of this prescription had been made in her medical chart.

The patient and her family were informed of the role of metoclopramide in her agitation, she was advised to stop the drug, and was discharged. That same evening, however, she was readmitted with anxiety and suicidal ideation. She received temazepam and one dose of haloperidol $1 \mathrm{mg}$ 
(also a DA-antagonist!). The akathisia subsided in 4 days time and did not recur, while she continued on the same dose of benzodiazepines as before, but without metoclopramide and haloperidol. Afterwards, on genotyping, no variant alleles of cytochrome P450 2D6 with diminished or absent metabolic activity possibly predisposing for psychomotor side effects of metoclopramide were found [8].

\section{Discussion}

Neuroleptic-induced acute akathisia is defined as a combination of subjective complaints of restlessness (including amongst others dysphoria and anxiety) and at least one of the following observed movements: fidgety movements or swinging of the legs while seated, rocking from foot to foot or "walking on the spot" while standing, pacing to relieve the restlessness, or an inability to sit or stand still for at least several minutes. Akathisia usually develops within weeks after initiation or dose increase of a neuroleptic drug, can be severe and may be associated with dysphoria, irritability, aggression and suicide attempts [9].

All DA-receptor blocking drugs can cause akathisia and as all antipsychotic drugs impact on DA-receptors, akathisia is a frequently occurring side effect of antipsychotics. As a potent DA-receptor blocking drug, metoclopramide is associated with psychomotor side effects, most notably acute dystonias, akathisia and parkinsonism and has probably some efficacy as an antipsychotic [2, 3, 10].

In this case, in concordance with our overall experience with similar cases, it was quite difficult to convince the clinicians that the psychomotor side effects were in fact caused by metoclopramide, a drug that is commonly prescribed without causing much trouble. This generally benign side effect profile might explain that the awareness of clinicians of psychomotor side effects of metoclopramide is sometimes lacking.

\section{Conclusion}

This patient displayed the classical syndrome of akathisia as a probable adverse effect of metoclopramide and highlights the psychomotor side effects of metoclopramide once more. It also illustrates the importance of routinely incorporating side effects of drugs in the differential diagnosis, also when alternative explanations (such as difficulties of the patient to adjust psychologically to disease) appear plausible.

\section{Conflicts of interest None.}

Open Access This article is distributed under the terms of the Creative Commons Attribution Noncommercial License which permits any noncommercial use, distribution, and reproduction in any medium, provided the original author(s) and source are credited.

\section{References}

1. Matok I, Gorodischer R, Koren G, Sheiner E, Wiznitzer A, Levy A. The safety of metoclopramide use in the first trimester of pregnancy. N Engl J Med. 2009;360(24):2528-35.

2. Pasricha PJ, Pehlivanov N, Sugumar A, Jankovic J. Drug insight: from disturbed motility to disordered movement-a review of the clinical benefits and medicolegal risks of metoclopramide. Nat Clin Pract Gastroenterol Hepatol. 2006;3:138-48.

3. Dingli K, Morgan R, Leen C. Tetanus versus acute dystonic reaction caused by metoclopramide. Br Med J. 2007;334: 899-900.

4. Bateman DN, Rawlins MD, Simpson JM. Extrapyramidal reactions with metoclopramide. Br Med J. 1985;291:930-2.

5. Miller LG, Jankovic J. Metoclopramide-induced movement disorders. Arch Intern Med. 1989;149:2486-92.

6. Ganzini L, Casey DE, Hoffman WF, McCall AL. The prevalence of metoclopramide-induced tardive dyskinesia and acute extrapyramidal movement disorders. Arch Intern Med. 1993;153:1469-75.

7. Bijl D. Metoclopramide en bewegingsstoornissen. Geneesmiddelen Bull. 2009;43:114.

8. Van der Padt A, van Schaik RHN, Sonneveld P. Acute dystonic reaction to metoclopramide in patients carrying homozygous cytochrome P450 2D6 genetic polymorphisms. Neth J Med. 2006;64:160-2.

9. Wright MT. Antiemetics, akathisia and pregnancy. Psychosomatics. 2007;48:461-6.

10. Stanley M, Lautin A, Rotrosen J, Gershon S, Kleinberg D. Metoclopramide: antipsychotic efficacy of a drug lacking potency in receptor models. Psychopharmacology (Berl). 1980;71: 219-25. 